\title{
25 Research Square \\ Effect of Different Types of Oil Intake on the Blood Index and the Intestinal Flora of Rats
}

\section{Yan Xu}

Yangzhou University College of food science and engineering

\section{Wenzheng Zhu}

Yangzhou University

\section{Qingfeng Ge}

Yangzhou University

\section{Xiaoyan Zhou ( $\approx$ yzuxyz@163.com )}

School of Food Science and Engineering, Yangzhou University, No.196 Huayang West Road, Hanjiang District, Yangzhou City, Jiangsu Province 225127, China

\section{Original article}

Keywords: Growing rats, Different oil, Blood lipids, 16S rRNA sequencing, Gut microbiota

Posted Date: May 13th, 2021

DOI: https://doi.org/10.21203/rs.3.rs-493907/v1

License: (9) (1) This work is licensed under a Creative Commons Attribution 4.0 International License. Read Full License 


\section{Abstract}

The effect of different oil-based diets on the blood index and the gut microbiota of rats was investigated. Rats in sample treatment groups were fed with stewed lard, refined lard, fish oil and soybean oil at a normal dose of $16.9 \%$ for 6 weeks. Compared with refined lard group, stewed lard treatment group showed reduced fasting blood sugar and blood lipid levels and improved nutrient absorption capacity of the intestine. The blood indexes of glucose (Glu), total cholesterol (TC) and total triglyceride (TG) in fish oil treatment group were relatively low. The abundance of Bacteroidetes in the fat-free group decreased, and the abundance of Firmicutes increased. The Firmicutes/Bacteroidetes ratio of the fish oil group was relatively low, and the Firmicutes/Bacteroidetes ratio of the stewed lard group and the soybean oil group was lower than that of the refined lard group. The abundance of Bacteroidaceae in the stewed pork fat group was increased. Research results show that fat-free diets will increase the risk of obesity to a certain extent; compared with refined lard, stewed lard, soybean oil and fish oil can reduce the risk of obesity to a certain extent; the addition and types of dietary fat will affect the abundance and diversity of rat intestinal flora.

\section{Introduction}

Dietary fat is an important part of human diet, and its nutritional value depends to a large extent on the composition of fatty acids, which is closely related to human health (Roche et al., 2014). At present, epidemiological studies mainly focus on the side effects of saturated fat and the positive effects of unsaturated fatty acids. Studies have shown that the consumption of saturated fat is positively correlated with the mortality of coronary heart disease, and other studies believe that the consumption of fish oil is negatively correlated with the mortality of coronary heart disease (Krisetherton et al., 2002). However, the existing studies have not taken into account the body's real needs for fat, and most of them are short-term studies based on high fat or disease models(Gauze-Gnagne et al., 2020; Adil et al., 2018). Lard and soybean oil are commonly used edible oils for Chinese residents, and fish oil is the most studied health oil. Stewed lard is obtained by simmering pork belly in low heat for a long time to extract fat. Compared with refined lard, the saturated fatty acid (SFA) of stewed lard decreased, and the monounsaturated fatty acid (MUFA) increased, and the content of other fatty acids also changed accordingly. The fatty acid composition of stewed lard is different from refined lard, fish oil, and soybean oil: refined lard has a high content of SFA and MUFA, fish oil is rich in n-3 PUFA such as EPA and DHA, and soybean oil is rich in n-6 PUFA.

Dietary fat can provide the body with essential fatty acids and is a carrier of fat-soluble vitamins. At the same time, it plays an important role in the body's fat metabolism, sugar metabolism and lipid peroxidation. Different types of fats have different effects on the body's growth performance and intestinal microbes. At present, there are relatively few studies on the effect of dietary fat on the intestinal microbes of the body, and most studies have shown that high-fat diets can disrupt the intestinal microflora of the body (Zhang et al., 2012; Ghosh S et al., 2013). It was found that diets with different fat content can regulate the function of gut microbes in rats before and after weaning (Z S and Bujňáková, 2017). Compared with a low-fat and high-carbohydrate diet, a high-fat and low-carbohydrate diet significantly reduces the concentration of short-chain fatty acids and the number of bifidobacteria in the body's feces (Brinkworth et al., 2009). Studies have shown that diets with different fat sources will have a certain impact on the microflora of the cecal contents of piglets (Li et al., 2017).Different effects of high-fat diets rich in different oils on lipid metabolism, oxidative stress was investigated, gut mirobiota and the high-fat diet exerted detrimental effects on the specific alterations in the gut microbiota, which increased the Actinobacteria and Enterococcaceae abundance, also decreased Bacteroidetes, Proteobacteria, Lactobacillales and microbiota diversity(Liu et al., 2021). 
At present, most of the research on intestinal microbes uses high-throughput technology, which has obvious advantages in throughput and high resolution compared with traditional molecular detection technology.

\section{Materials And Methods}

\section{Animals and diets}

All experiments were carried out in compliance with the relevant guidelines and regulations of the Ethical Committee of Experimental Animal Center of Yangzhou University. A total of 48 4-week-old female Wistar rats were housed in a specific pathogen-free animal center (SCXK (Jiangsu) 2017-0007, Yangzhou, Jiangsu, China). The temperature (24.0 $\left.\pm 0.5^{\circ} \mathrm{C}\right)$ and relative humidity $(60 \pm 10 \%)$ were kept constant during the experiment, with a 12-h light cycle. Rats were fed a standard chow diet during a 1-week acclimation period. Then, animals were assigned to one of five diet groups (eight rats in each group), that is, fat-free (WZ), stewed lard (SY), refined lard (JL), fish oil (YY) and soybean oil (DY) groups. Refined lard, fish oil and soybean oil were commercially obtained. Stewed lard is made by simmering pork belly for 120 min, removing the broth, chopped, freeze-dried, and then extracted according to the method of Folch (Folch et al., 1957). The stewed lard was placed in a reduced-pressure vacuum drying oven at $40^{\circ} \mathrm{C}$ for 48 hours to completely remove the remaining organic solvents, and vacuum packaged at $-20^{\circ} \mathrm{C}$ until use. The composition of fatty acids in different oil were shown in Table 1. The diets were prepared according to the AIN-93G diet formulation and the diet formulation was shown in Table 2. During the experiment, the feed of each rat was refreshed every day to prevent oxidative deterioration of fat in the feed.

Observe the rat's diet rule to ensure that the daily diet is basically free of excess food, and provide a certain basis for the feeding amount of each experimental group during the formal experiment. Body weight and feed intake of rats were routinely recorded for calculating the average daily gain.

\section{Sample collection}

Fresh feces were collected after rats were fed for 14 days. Normally, the animals excrete feces when they are hung by their tails. The fecal samples were immediately frozen in liquid nitrogen and then stored at $-80{ }^{\circ} \mathrm{C}$ until further analyses. At 6 weeks of feeding, the blood of rats were collected without fasting, the tubes stood at room temperature for $30 \mathrm{~min}$ and then were centrifuged at $3,000 \mathrm{~g}$ for $30 \mathrm{~min}$ to pellet the blood cells. Serum samples were collected and stored at $-80^{\circ} \mathrm{C}$. At 6 weeks, all the rats were euthanized by cervical dislocation. The liver, kidney, heart and spleen were taken and weighed. Relative weights of the liver, kidney, heart and spleen tissues were calculated according to body weight. The duodenum and jejunum tissues of rats were soaked in $4 \%$ paraformaldehyde solution for fixation.

\section{Serum biochemical indicators}

Serum biochemical indicators containing glucose(GLU), triglycerides (TG), total cholesterol (TC), high density lipoprotein cholesterol (HDL-C), and low density lipoprotein cholesterol (LDL-C) were determined using the commercially available kits from Nanjing Jiancheng Bioengineering Institute (Nanjing,China).

\section{S rRNA gene sequencing}

Total genomic DNA in fecal samples was extracted using the QIAamp DNA Stool Mini Kit (Qiagen 51 504, Dusseldorf, Nordrhein-westfalen, Germany) according to the manufacturer's instructions. The DNA was quantified by a Nanodrop ${ }^{\circledR}$ spectrophotometer (Nanodrop 2000, Thermo Fisher Scientific,Waltham, MA). Purified DNA was used 
to amplify the V4 region of $16 \mathrm{~S}$ rRNA, which is associated with the lowest taxonomic assignment error rate. All DNA samples were kept at $-20^{\circ} \mathrm{C}$ until sequencing. The V4-V5 hypervariable region of the $16 \mathrm{~S}$ ribosomal RNA gene was selected for amplification from DNA samples. Polymerase chain reaction (PCR) was performed in triplicate. Amplicons were extracted from $2 \%$ agarose gels and purified using the AxyPrep DNA Gel extraction kit (Axygen Biosciences, Union City, CA) according to the manufacturer's instructions and quantified using QuantiFluor ${ }^{\mathrm{TM}}-\mathrm{ST}$ (Promega, Madison, WI). The pooled DNA product was used to construct Illumina Pair-End library following Illumina's genomic DNA library preparation procedure. Then the amplicon library was double-end sequenced (2 $\times 300 \mathrm{bp}$ ) on an Illumina MiSeq platform (San Diego, CA) according to the standard protocols.

\section{Bioinformatics analysis}

Raw fastq files were trimmed and chimeric sequences were identified and removed from all samples to reduce noise, and operational taxonomic units (OTUs) were clustered with $\geq 97 \%$ similarity cutoff using QIIME. Then community richness estimator (Chao and ACE), diversity indices (Shannon and Simpson), and Good's coverage were calculated (Schloss et al., 2009).Duncan's multiple comparison was applied to compare averages between any two groups.

Differences were considered significant if $p$ values were $<0.05$. Principal coordinate analysis (PCoA) and clustering analysis were applied on the basis of the OTUs to offer an overview of the fecal microbial composition(Lozupone et al., 2011). Multivariate analysis of variance (MANOVA) was conducted to further confirm the observed differences. Linear discriminant analysis effect size (LEfSe) analysis (http://huttenhower.sph.harvard.edu/galaxy/) was carried out to discover biomarkers for fecal bacteria and to distinguish between biological conditions among different groups (Segata et al., 2011). The R (pheatmap package) and Cytoscape were applied to visualize the relationships through correlation heatmap and network diagrams respectively.

\section{Statistics analysis}

All of the data were generated using SPSS 16.0 software and are represented here as mean \pm standard deviation (SD).Differences between the mean values were evaluated using oneway analysis of variance and Tukey's multiple comparisons test (if applicable). A P value $<0.05$ was considered to be statistically significant.

\section{Results}

\section{Physical Characteristics}

During the experimental period, the rats in each group grew well, and there was no significant difference in initial body weight. It can be seen from Figure 1 that the body weight of the rats has increased, and there is no significant difference between the groups at each time point $(P>0.05)$. The experimental results show that under the condition of intake of nutritional energy, the addition and type of dietary fat has no significant effect on the body mass of rats $(P>0.05)$. Under the conditions of intake and other nutritional energy, the addition and types of dietary fat have no significant effect on the body mass of rats.

It can be seen from table 3 that dietary fat has no significant effect on the heart index, spleen index and kidney index of rats in each group, indicating that the addition and type of dietary fat has no effect on rat kidney, heart and spleen. The liver index of JL group was significantly higher than that of rats in WZ, YY and DY groups $(P<0.05)$.

\section{Blood biochemical indicators}


As is shown in Table 4, compared with the JL group, the serum GLU content of rats in the SY group and the DY group was relatively low, but the difference was not significant. The fasting blood glucose value (GLU) of rats in the YY group was lower than that of the WZ group, and significantly lower than other dietary fat mixed diet groups $(P<$ 0.05). Compared with the JL group, the serum total cholesterol (TC) content of the SY group was relatively lower. The serum TC content of rats in the YY group was significantly lower than that in the WZ group and other dietary fat mixed diet groups $(P<0.05)$. Serum triglyceride $(T G)$ levels in WZ and $Y Y$ groups were significantly lower than in SY, $\mathrm{JL}$ and DY groups $(P<0.05)$. Compared with refined lard, stewed lard can reduce the content of TC and TG in rat serum to a certain extent. The high-density lipoprotein (HDL-C) of WZ and DY groups was significantly higher than that of SY, JL and YY groups $(P<0.05)$. The HDL-C content of $Y Y$ group was lower than that of other groups. The level of low-density lipoprotein (LDL-C) in the JL group was significantly higher than that in the YY and DY groups ( $P$ $<0.05)$. The serum HDL-C level of the SY group was higher than that of the JL lard group and the LDL-C level was lower than that of the $\mathrm{JL}$ group, but the difference was not significant $(P>0.05)$.

\section{Morphology of rat intestine}

As is shown in Fig.2 and Table 5, the villus height of the homozygous diet supplemented with dietary fat was significantly higher than that of the fat-free group $(P<0.05)$. The increase in villus height helps to improve the intestinal health of rats, thereby increasing their digestion and absorption of nutrients ability. The depth of the crypt reflects the rate of intestinal villi forming epithelial cells, and the shallower crypt indicates that the maturation rate of intestinal epithelial cells has increased. The villi and crypts in the intestinal tract of rats in the homozygous diet supplemented with dietary fat grew better, and their digestive physiological functions were better than those in the WZ group, which helped the growth of animals. The intestinal wall thickness of the WZ group was significantly lower than that of other homozygous diets supplemented with dietary fat $(P<0.05)$. The ratio of villus length/crypt depth in the WZ group was significantly lower than that of other homozygous diets supplemented with dietary fat $(P<$ 0.05). The ratio of villus height/crypt depth in the SY group was higher than that of the other groups, it shows that the intake of a certain amount of stewed lard can increase the villus height and crypt depth of the duodenum and jejunum, and enhance the digestion and absorption function of the intestine.

\section{Cholesterol and triglyceride content in rat liver}

As is shown in Fig. 3, it is found that the cholesterol (TC) and triglyceride (TG) levels in the liver of the fish oil group were significantly lower than those of the other homozygous diets supplemented with dietary fat $(P<0.05)$. Fish oil not only significantly reduced the blood lipid content in rat serum, but also significantly reduced the cholesterol and triglyceride content in the liver.

\section{Richness and diversity analyses}

The community richness estimators (Chao and ACE), and diversity indices (Shannon and Simpson) were calculated in order to evaluate the alpha diversity (Table 6). The Chao1 and ACE indexes of the SY group were higher than other experimental groups. Compared with other dietary fat mixed diet groups, the Chao1 and ACE indexes of the WZ group were relatively low, and the Shannon index was relatively low. Experimental results show that stewed lard can increase the abundance of intestinal flora to a certain extent, while fat-free diet will reduce the abundance and diversity of intestinal flora.

\section{Rat Fecal Microbe Composition}


At the phylum level, Bacteroidetes and Firmicutes were predominant in all samples. In the WZ group, Bacteroidetes was the most abundant phylum, accounting for $48.00 \%$, followed by Firmicutes and Proteobacteria accounting for $44.11 \%$ and $3.37 \%$ respectively. In the SY group, Bacteroidetes was the most abundant phylum, accounting for $54.50 \%$, followed by Firmicutes and Proteobacteria accounting for $42.47 \%$ and $1.22 \%$, respectively. In the JL group, Bacteroidetes was the most abundant phylum, accounting for $51.20 \%$, followed by Firmicutes and Proteobacteria accounting for $43.76 \%$ and $2.11 \%$, respectively. In the $Y Y$ group, Bacteroidetes was the most abundant phylum, accounting for $67.74 \%$, followed by Firmicutes and Proteobacteria, accounting for $29.09 \%$ and $1.74 \%$, respectively. In the DY group, Bacteroidetes was the most abundant phylum, accounting for $54.93 \%$, followed by Firmicutes and Proteobacteria accounting for $37.65 \%$ and $2.11 \%$, respectively. It can be seen from Fig. 4 that the dominant bacteria attached to the WZ group, SY group, JL group, YY group and DY group are basically the same at the phylum level, and there are significant individual differences among all samples. As is shown in Table 7, the proportion of Bacteroidetes in each sample ranges from $41.52 \%$ to $77.38 \%$, and the proportion of Firmicutes in each sample ranges from $20.90 \%$ to $48.52 \%$. The proportion of Proteobacteria in each sample ranges from $0.71 \%$ to $6.20 \%$. It can be seen from Table 7 that the ratio of Firmicutes/Bacteroidetes (F/B) in the SY group is 0.79 lower than that of the $\mathrm{JL}$ group. Compared with the fat-added diet group, the abundance of Bacteroidetes in the WZ group decreased, and the abundance of Firmicutes increased. The F/B ratio of the WZ group was the highest at 0.92. In addition, the abundance of Bacteroidetes in the YY group was significantly higher than that in the fat-free group $(P<0.05)$, the abundance of Firmicutes in the YY group was significantly lower than that in the SY, JL, and WZ groups $(P<0.05)$, and the lowest $F / B$ ratio in the $Y Y$ group is 0.44 .

As is shown in Fig. 5 and Table 8, the abundance of Bacteroidaceae in the SY group was 29.95\% higher than the other groups, and was significantly higher than that in the $J L$ group $(P<0.05)$. There was no significant difference in

the abundance of Bacteroidaceae in the WZ, YY and DY groups. The abundance of S24-7 in the WZ group was lower than that of other dietary fat mixed diet groups, and significantly lower than that of the JL group $(P<0.05)$. The abundance of S24-7 in SY group was not significantly different from WZ, YY and DY groups. The highest abundance of Ruminococcaceae in the WZ group was $19.82 \%$, and the lowest abundance of Ruminococcaceae in the YY group was $12.60 \%$.

\section{Discussion}

The weight of rats in the fat-free group was lower than that in the homozygous diet group with added dietary fat, and the experimental results were similar to the results of previous studies. The liver is a key organ for the metabolism of various nutrients. Lipid metabolism is inseparable from the liver. The liver is the main place for the metabolism of intermediate products. Once lipid metabolism disorder occurs, excess lipids are easy to accumulate in the liver and cause fatty liver disease (Kang et al., 2021). The liver index of SY group was not significantly different from that of WZ and DY groups $(P>0.05)$. Our present results showed that compared with refined lard, stewed lard has a lower probability of liver metabolism disorders or liver function damage.

Dietary fat has different effects on the serum indexes of rats. Compared with refined lard, stewed lard can reduce the content of GLU, TC, TG, LDL-C in the serum to a certain extent, and increase the content of HDL-C. Fish oil is rich in n3 PUFAs, especially EPA and DHA, which can improve the body's metabolic parameters (such as blood sugar, cholesterol and triglycerides) and reduce the accumulation of lipids in the carcass (Gondim et al., 2018). The atherosclerosis index of the refined lard group was significantly higher than that of the other groups, and the liver index was also higher than that of the other groups (Tung et al., 2019). The decrease in HDL-C levels in the YY group may be due to the richness of EPA and DHA in fish oil. As PUFA has no selective lipid-lowering effect, it reduces the 
level of HDL-C while reducing the TC level (Breetha et al., 2018). The serum TC content of rats fed fish oil was significantly lower than that of fat-free and other fat groups, which is consistent with related literature reports (Gondim et al., 2018; Harari et al., 2020). In contrast, refined lard tends to increase the level of LDL-C. Tung (Tung et al., 2019)found that excessive intake of refined lard rich in SFA will increase serum TC, TG and LDL-C levels and reduce HDL-C levels, while intake of tea seed oil rich in MUFA will reduce blood lipid levels.

The gut is a complex and dynamic ecosystem. The temporal pattern of microbial survival is the key for finding out core members responding to environmental changes. Many studies have shown that the composition of fecal microbiota is highly correlated with the colonic lumen and mucosa and moderately correlated with the distal small intestine (Yasuda et al., 2015). The small intestine is the main part for animals to digest and absorb nutrients, and the function of the digestive tract is closely related to the growth state of the animal (Xu et al., 1994). Small intestinal villi is the main tissue for nutrient absorption, and its height can reflect the mitotic activity of intestinal crypt cells. Studies have shown that changes in villi height can affect the surface area of intestinal villi, thereby affecting the absorption of nutrients (Czech et al., 2020; Caspary et al., 1992). Nutritional physiology studies have shown that the ratio of villi length/crypt depth can comprehensively reflect the physiological functions of the intestine. An increase in the ratio indicates an increase in the digestion and absorption function of the intestine, and a decrease in the ratio indicates a decline in the digestion and absorption function (Rehman et al., 2018). The villus height of the homozygous diet supplemented with dietary fat was significantly higher than that of the fat-free group, and the ratio of villus height/crypt depth in the experimental group was higher than that of the other groups of rats, indicating that the consumption of stewed pork fat can enhance intestinal digestion and energy absorption. It can be seen that a fat-free diet can reduce fasting blood sugar and blood lipid levels to a certain extent, but it also reduces the intestinal nutrient absorption capacity. Compared with the refined lard group, stewed lard can reduce the fasting blood sugar and blood lipid levels of the body to a certain extent, and can improve the intestinal nutrient absorption capacity.

Fish oil can significantly down-regulate the expression of fatty acid synthesis-related enzymes G6PDH, ME, FAS and other mRNA, and can increase the mRNA expression of fatty acid decomposing enzymes CPT-1 and CPT-2 in the rat liver, resulting in a decrease in liver and serum lipid concentrations(Godea et al., 2020). The content of TC and TG in the liver of the refined lard group was higher than that of the fat-free group and other homozygous diet groups with added dietary fat. Wang xiaoke(Wang et al., 2013) compared the occurrence of obesity induced by high-fat diet with different oil components and found that the liver lipid content of the lard group was significantly higher than that of the soybean oil group. It is speculated that the type of fat is responsible for inducing lipid production and promoting lipid breakdown. The expression of related factors is related to the different utility. The results of this study show that compared with refined lard, stewed lard has lower levels of TC and TG in the liver.

Firmicutes and Bacteroides are the most dominant bacterial phyla in the body, which can affect the energy extraction efficiency of the host and are related to the host's excessive obesity. Obese animals or humans have a higher abundance of Firmicutes in the gut flora (Kallus et al., 2012). In addition, many studies have found that obesity induced by high-fat diet will increase the ratio of Firmicutes to Bacteroides, which affects the metabolic function of intestinal microbes and increases their energy intake (Turnbaugh et al., 2006; Power et al., 2014; Smits et al., 2013). From the changes in the abundance of Bacteroidetes and Firmicutes, it can be proved that fish oil has a certain inhibitory effect on obesity and the beneficial effects of eating dietary fat on the intestinal flora, refined lard is more likely to cause obesity than stewed lard. 
The composition of the intestinal microbial colonies of five groups of rat fecal samples and the distribution and abundance of bacterial species were analyzed. The abundance of Bacteroidaceae in the SY group was higher than the other groups. The experimental results show that the stewed lard can significantly increase the abundance of Bacteroidaceae in the body. The abundance of Bacteroides in the intestinal flora of obese rats induced by high-fat diet was lower than that in the normal group, green tea polyphenols can significantly increase the relative abundance of Bacteroides and Proteus after 3 weeks of intervention in rats, thereby reducing obesity in rats induced by high-fat diet(Guo et al., 2017).

S24-7 under the phylum Bacteroides has not been isolated and cultured in vitro, and its biological characteristics are not yet clear. Ormerod (Ormerod et al., 2016) found that S24-7 is the main member of the gut microbiota and is highly present in the gastrointestinal tract of warm-blooded animals. They named it "Candidatus Homeothermaceae" and speculated that it belongs to Gram-negative anaerobes that can ferment carbohydrates extensively. Partial hepatectomy resulted in a significant increase in S24-7 of the intestinal flora Bacteroides phylum and a significant decrease in Firmicutes, Lachnospiraceae and Ruminococcaceae (Liu et al., 2016).It is also suggested that liver metabolism and immune function are closely related to the abundance of Lachnospiraceae, Ruminococcacea and S24-7.The results show that fat-free diet will reduce the abundance of S24-7 in the body to a certain extent. Rumen cocci mainly exist in the rumen, cecum and large intestine of mammals. Studies have shown that the rumen cocci in the intestine of high-fat rats will significantly decrease (Daniel et al., 2014), and the intake and types of dietary fat will have a certain impact on the relative abundance of Ruminococcaceae in the body. The abundance of Lachnospiraceae, Desulfovibrionaceae, Acidaminococcaceae, Coriobacteriaceae and Bilophila is positively correlated with obesity (Zhao et al., 2017). The experimental results showed that compared with the dietary fat group, the abundance of Lachnospiraceae in the WZ group was relatively higher, and the abundance of Lachnospiraceae in the SY group was lower than that in the JL group. It shows that fat-free diet may also lead to obesity, refined lard is more likely to produce obesity than stewed lard.

Our study revealed that fat-free diet can reduce fasting blood sugar and blood lipid levels to a certain extent, but it also reduces the intestinal nutrient absorption capacity. Compared with the refined lard group, stewed lard can reduce the body's fasting blood sugar and blood lipid levels to a certain extent, and can improve the intestinal nutrient absorption capacity. Compared with other dietary fat groups, stewed pork fat will increase the abundance of Bacteroidaceae in rats, and fat-free diet will decrease the abundance of S24-7in rats. It shows that the addition and types of dietary fat will affect the distribution of rat intestinal flora to a certain extent. Therefore, we can try to choose some fats with higher unsaturated fatty acid content which similar to fish oil, and at the same time properly intake stewed lard to improve the abundance of intestinal flora.

\section{Abbreviations}

WZ: fat-free ; SY: stewed lard; JL: refined lard; YY: fish oil ; DY: soybean oil ; Glu : glucose; TC: total cholesterol; TG: total triglyceride; SFA : saturated fatty acid; MUFA monounsaturated fatty acid ; HDL-C: high-density lipoprotein ; LDL-C: low-density lipoprotein; F/B: the ratio of Firmicutes/Bacteroidetes

\section{Declarations}

\section{Ethics approval and consent to participate}

All animal procedures were performed in accordance with the Guidelines for Care and Use of Laboratory Animals of Yangzhou University. 


\section{Consent for publication}

All authors approved the final version of the manuscript and consent for publication

\section{Availability of data and materials}

All data generated or analysed during this study are included in this published article.

\section{Competing interests}

The authors declare that they have no competing interests

\section{Authors' contributions}

$\mathrm{YX}$, as the lead contributor, contributed in designing and conducting the experiments, collecting the data and summarizing the literatures. WZ assisted the lead contributor to conduct the experiments and revise the manuscript. QG and XZ provided technical guidance and modified the manuscript. XZ, as the corresponding author, provided financial support and contributed in organizing, writing, editing and revising the manuscript. All authors reviewed the manuscript. All authors read and approved the manuscript.

\section{Acknowledgements}

Not applicable.

\section{Funding}

Not applicable

\section{References}

1. Adil H, Nadia M, Virginie M, Lionel U, Hafida M, Fabienne PV, Nezha A, Gérard T ( 2018) A comparative study on the effect of argan oil vs fish oil on risk factors for cardio-vascular disease in high-fat-fed rats. Nutrition 57:3239. https://doi.org/10.1016/j.nut.2018.05.027

2. A.M. DF, Corrado DN, Arnaldo A (1994) Self-organizing multisensor systems for odour classification: internal categorization, adaptation and drift rejection. Elsevier 18:244-258. https://doi.org/10.1016/09254005(94)87090-x

3. Breetha R, Ravichandra TR(2018) Dietary Unsaturated Fatty Acids Modulate Maternal Dyslipidemia-Induced DNA Methylation and Histone Acetylation in Placenta and Fetal Liver in Rats. Lipids 53:581-588. https://doi.org/10.1002/lipd.12074

4. Brinkworth GD, Noakes M, Clifton PM, Bird AR (2009) Comparative effects of very low-carbohydrate, high-fat and high-carbohydrate, low-fat weight-loss diets on bowel habit and faecal short-chain fatty acids and bacterial populations. Br J Nutr 101(10):1493-1502. https://doi.org/10.1017/S0007114508094658

5. Caspary WF(1992) Physiology and pathophysiology of intestinal absorption. American Journal of Clinical Nutrition 55(1):299S-308S. https://doi.org/10.1093/ajcn/55.1.299s

6. Czech A, Sembratowicz I, Zieba G(2020) Effect of the use of Yarrowia lipolytica and Saccharomyces cerevisiae yeast with a probiotic in the diet of turkeys on their gut microbiota and immunity. Veterinární Medicína 65(4):174-82. https://doi.org/10.17221/145/2019-vetmed 
7. Daniel H, Gholami AM, Berry D, Desmarchelier C, Hahne H, Loh G, Mondot S, Lepage P, Rothballer M, Walker A, Böhm C, Wenning M, Wagner M, Blaut M, Schmitt-Kopplin P, Kuster B, Haller D, Clavel T(2014) High-fat diet alters gut microbiota physiology in mice. Isme Journal 8(2):295-308. https://doi.org/10.1038/ismej.2013.155

8. Folch J, Lees M, Sloane GS (1957) A simple method for the isolation and purification of total lipides from animal tissues. Journal of Biological Chemistry 1957,226(1):497-509. https://doi.org/10.1016/s00219258(18)64849-5

9. Gauze-Gnagne C, Raynaud F, Djohan YF, Lauret C, Feillet-Coudray C, Coudray C, Monde A, Koffi G, Morena M, Camara-cisse M, Cristol JP, Badia E ( 2020) Impact of diets rich in olive oil, palm oil or lard on myokine expression in rats.Food \& Function 11(10):9114-9128. https://doi.org/10.1039/d0fo01269f

10. Ghosh S, Molcan E, Decoffe D, Dai C, Gibson DL (2013) Diets rich in n-6 PUFA induce intestinal microbial dysbiosis in aged mice. British Journal of Nutrition 110(3):515-523.

https://doi.org/10.1017/s0007114512005326

11. Godea S, Ciubotariu D, Danciu M, Lupușoru RV, Ghiciuc CM, Cernescu I, Gheţu N, Lupei M, Lupușoru C(2020) Improvement in serum lipids and liver morphology after supplementation of the diet with fish oil is more evident under regular feeding conditions than under high-fat or mixed diets in rats. Lipids in Health and Disease 19(1):162. https://doi.org/10.1186/s12944-020-01339-y

12. Gondim PN, Rosa PV, Okamura D, Silva VO, Andrade EF, Biihrer DA, Pereira L (2018) Benefits of Fish Oil Consumption over Other Sources of Lipids on Metabolic Parameters in Obese Rats.Nutrients 10(1):65. https://doi.org/10.3390/nu10010065

13. Guo X, Cheng M, Zhang X, Cao J, Wu Z, Weng P(2017) Green tea polyphenols reduce obesity in high-fat dietinduced mice by modulating intestinal microbiota composition. International Journal of Food Science \& Technology 52(8):1723-1730. https://doi.org/10.1016/s0016-5085(13)60325-x

14. Harari A, Frenkel AL, Barshack I, Sagee A, Cohen H, Kamari Y, Harats D, Kfir MK, Shaish A (2019) Addition of fish oil to atherogenic high fat diet inhibited atherogenesis while olive oil did not, in LDL receptor KO mice ScienceDirect. Nutrition, Metabolism and Cardiovascular Diseases 30(4):709-716.

https://doi.org/10.1016/j.numecd.2019.12.007

15. HI A, Hza B , Hui XA. , Xian YA, Ly A, Sw A, Jw C, Gs A (2020) Different effects of high-fat diets rich in different oils on lipids metabolism, oxidative stress and gut microbiota. Food Research International 141:1-15. https://doi.org/10.1016/j.foodres.2020.110078

16. Kang K, Sun Y, Pan D, Sang LX, Chang B (2021). Distinctive gut microbial dysbiosis between chronic alcoholic fatty liver disease and metabolicassociated fatty liver disease in mice. Experimental and Therapeutic Medicine 21(5). https://doi.org/10.3892/etm.2021.9862

17. Kallus SJ, Brandt LJ(2012) The intestinal microbiota and obesity. Journal of Clinical Gastroenterology 46(1):16. https://doi.org/10.1097/mcg.0b013e31823711fd

18. Kashyap PC, Marcobal A, Higginbottom S, Smits SA, Sonnenburg J(2013) Individualized Responses of Gut Microbiota to Dietary Intervention Modeled in Humanized Mice. Gastroenterology 144(5):S-88. https://doi.org/10.1016/s0016-5085(13)60325-x

19. Krisetherton PM, Harris WS, Appel LJ (2002) Fish consumption, fish oil, omega-3 fatty acids, and cardiovascular disease. Circulation 106(2):2747-2757. https://doi.org/10.1016/b978-0-12-802844-5.00007-5

20. Li H, Zhu Y, Zhao F, Song S, Li C (2017) Fish oil, lard and soybean oil differentially shape gut microbiota of middle-aged rats. Scientific Reports 7(1):826. https://doi.org/10.1038/s41598-017-00969-0 
21. Liu HX, Rocha CS, Dandekar S, Wan YJY(2016) Functional analysis of the relationship between intestinal microbiota and the expression of hepatic genes and pathways during the course of liver regeneration. Journal of Hepatology 64(3):641-50. https://doi.org/10.1016/j.jhep.2015.09.022

22. Lozupone C, Lladser M, Knights D, Stombaugh J, Knight R(2011) UniFrac: An effective distance metric for microbial community comparison. The ISME journal 5:169-172. https://doi.org/10.1038/ismej.2010.133

23. Ormerod KL, Wood DLA, Lachner N, Gellatly SL, Daly JN, Parsons JD, Dal'Molin CG.O, Palfreyman RW, Nielsen LK, Cooper MA, Morrison M, Hansbro PM, Hugenholtz P (2016) Genomic characterization of the uncultured Bacteroidales family S24-7 inhabiting the guts of homeothermic animals. Microbiome 4(1):36. https://doi.org/10.1186/s40168-016-0181-2

24. Power SE, O'Toole PW, Stanton C, Ross RP, Fitzgerald GF(2014)Intestinal microbiota, diet and health[J].British Journal of Nutrition 111(3):387-402. https://doi.org/10.1201/b17254-19

25. Rehman ZU, Che L, Ren S, Liao Y, Qiu X, Yu S, Sun Y, Tan L, Song C, Liu W, Ding Z, Munir M, Nair V, Meng C, Ding C (2018) Supplementation of Vitamin E Protects Chickens from Newcastle Disease Virus-Mediated Exacerbation of Intestinal Oxidative Stress and Tissue Damage. Cellular Physiology and Biochemistry 47:16551666. https://doi.org/10.1159/000490984

26. Roche E, Ramíreztortosa CL, Arribas MI, Ochoa JJ, Sirventbelando JE, Battino M, Ramírez-Tortosa MC, González-Alonso A, Pérez-López, MP, Quiles JL (2014) Comparative analysis of pancreatic changes in aged rats fed life long with sunflower, fish, or olive oils. Journals of Gerontology 69(8):934-944.

https://doi.org/10.1093/gerona/glt157

27. Schloss PD, Westcott SL, Ryabin T, Hall JR, Hartmann M, Hollister EB, Lesniewski RA, Oakley BB, Parks DH, Robinson CJ, Sahl JW, Stres B, Thallinger GG, Van Horn DJ, Weber CF(2009) Introducing mothur: open-source, platform-independent, community-supported software for describing and comparing microbial communities. Applied and environmental microbiology 75(23):7537-41. https://doi.org/10.1128/aem.01541-09

28. Segata N, Izard J, Waldron L, Gevers D, Miropolsky L, Garrett WS, Huttenhower C (2011) Metagenomic biomarker discovery and explanation[J].Genome Biology 12(6):R60. https://doi.org/10.1186/gb-2011-12-6-r60

29. Tung YT, Hsu YJ, Chien YW, Huang CC, Huang WC, Chiu WC (2019)Tea Seed Oil Prevents Obesity, Reduces Physical Fatigue, and Improves Exercise Performance in High-Fat-Diet-Induced Obese Ovariectomized Mice. Molecules 24(5):980. https://doi.org/10.3390/molecules24050980

30. Turnbaugh PJ, Ley RE, Mahowald MA, Magrini V, Mardis ER, Gordon JI(2006)An obesity-associated gut microbiome with increased capacity for energy harvest. Nature 444(7122):1027-1031.

https://doi.org/10.1038/nature05414

31. Wang X, Cheng M, Zhao M, Ge A, Guo F, Zhang M, Zhang M, Yang Y, Liu L, Yang N (2013)Differential effects of high-fat-diet rich in lard oil or soybean oil on osteopontin expression and inflammation of adipose tissue in dietinduced obese rats. European Journal of Nutrition 52(3):1181-1189. https://doi.org/10.1007/s00394-012-0428z

32. Xu RJ, Mellor DJ, Birtles MJ, Breier BH, Gluckman PD(1994) Effects of oral IGF-I or IGF-II on digestive organ growth in newborn piglets. Biol Neonate 66(5):280-287. https://doi.org/10.1159/000244118

33. Yasuda K, Oh K, Ren B, Tickle TL, Franzosa EA, Wachtman LM, Miller AD, Westmoreland SV, Mansfield KG, Vallender EJ., Miller GM, Rowlett JK, Gevers D, Huttenhower C, Morgan XC (2015) Biogeography of the intestinal mucosal and lumenal microbiome in the rhesus macaque. Cell host \& microbe 17(3):385-391.

https://doi.org/10.1016/j.chom.2015.01.015

Page $11 / 21$ 
34. Zhao L, Zhang Q, Ma W, Tian F, Shen H, Zhou M(2017) A combination of quercetin and resveratrol reduces obesity in high-fat diet-fed rats by modulation of gut microbiota. Food \& Function 8(12):4644-4656.

https://doi.org/10.1039/c7fo01383c

35. Zhang C, Zhang M, Pang X, Zhao Y, Wang L, Zhao L ( 2012) Structural resilience of the gut microbiota in adult mice under high-fat dietary perturbations. Isme Journal 6(10):1848-1857.

https://doi.org/10.1038/ismej.2012.27

36. Z Š, Bujňáková D (2017) Effect of pre- and post-weaning high-fat dietary manipulation on intestinal microflora and alkaline phosphatase activity in male rats. Physiological Research 66(4):677-685.

https://doi.org/10.33549/physiolres.933500

\section{Tables}

Table 1 Fatty acid composition of experimental oils $[\mathrm{g} / 100 \mathrm{~g}$ oill 


\begin{tabular}{|c|c|c|c|c|}
\hline Fatty acid & SY & $\mathrm{JL}$ & DY & $Y Y$ \\
\hline C12:0 & 0.08 & 0.11 & 0.00 & 0.00 \\
\hline C14:0 & 1.55 & 1.76 & 0.07 & 1.13 \\
\hline C15:0 & 0.02 & 0.00 & 0.00 & 0.41 \\
\hline C16:0 & 25.85 & 24.79 & 10.89 & 20.64 \\
\hline C17:0 & 0.11 & 0.25 & 0.05 & 1.15 \\
\hline C18:0 & 11.34 & 15.25 & 4.86 & 6.72 \\
\hline C19:0 & 0.01 & 0.00 & 0.00 & 0.00 \\
\hline C20:0 & 0.22 & 0.22 & 0.41 & 1.26 \\
\hline C14:1 & 0.03 & 0.04 & 0.01 & 0.02 \\
\hline C16:1 & 3.51 & 2.55 & 2.11 & 0.12 \\
\hline C17:1 & 0.15 & 0.15 & 0.03 & 0.36 \\
\hline C18:1 & 44.65 & 37.81 & 22.44 & 27.78 \\
\hline C19:1 & 0.06 & 0.00 & 0.00 & 0.15 \\
\hline C20:1 & 1.04 & 0.81 & 0.17 & 2.97 \\
\hline C16:2 & 0.02 & 0.00 & 0.00 & 0.18 \\
\hline C18:2 & 10.64 & 15.65 & 52.61 & 17.48 \\
\hline C20:2 & 0.41 & 0.61 & 0.00 & 0.25 \\
\hline C18:3 & 0.01 & 0.00 & 6.35 & 0.30 \\
\hline C20:3 & 0.09 & 0.00 & 0.00 & 2.61 \\
\hline C20:4 & 0.13 & 0.00 & 0.00 & 0.11 \\
\hline C20:5 & 0.03 & 0.00 & 0.00 & 15.79 \\
\hline$C 22: 4$ & 0.05 & 0.00 & 0.00 & 0.57 \\
\hline C22:6 & 0.00 & 0.00 & 0.00 & 12.68 \\
\hline SFA & 39.18 & 42.38 & 16.28 & 31.31 \\
\hline MUFA & 49.44 & 41.36 & 24.76 & 31.40 \\
\hline PUFA & 11.38 & 16.26 & 58.96 & 37.29 \\
\hline UFA & 60.82 & 57.62 & 83.72 & 68.69 \\
\hline UFA/SFA & 1.55 & 1.36 & 5.14 & 2.19 \\
\hline
\end{tabular}




\begin{tabular}{|c|c|c|c|c|c|}
\hline Component (g/kg) & WZ & SY & $\mathrm{JL}$ & YY & DY \\
\hline Casein & 180.35 & 200.0 & 200.0 & 200.0 & 200.0 \\
\hline Corn starch & 519.82 & 397.49 & 397.49 & 397.49 & 397.49 \\
\hline Dextrin & 119.03 & 132.0 & 132.0 & 132.0 & 132.0 \\
\hline Sucrose & 90.17 & 100.0 & 100.0 & 100.0 & 100.0 \\
\hline Stewed lard & - & 70.0 & - & - & - \\
\hline Refined lard & - & - & 70.0 & - & - \\
\hline Fish oil & - & - & - & 70.0 & - \\
\hline Soybean oil & - & - & - & - & 70.0 \\
\hline Cellulose & 45.09 & 50.0 & 50.0 & 50.0 & 50.0 \\
\hline Minerals & 31.56 & 35.0 & 35.0 & 35.0 & 35.0 \\
\hline Vitamins & 9.02 & 10.0 & 10.0 & 10.0 & 10.0 \\
\hline L-cystine & 2.71 & 3.0 & 3.0 & 3.0 & 3.0 \\
\hline Choline Chloride & 2.25 & 2.5 & 2.5 & 2.5 & 2.5 \\
\hline Tert-butyl hydrogen & 0.01 & 0.01 & 0.01 & 0.01 & 0.01 \\
\hline \multicolumn{6}{|l|}{ Nutritional level } \\
\hline Total energy $₫ \mathrm{Kcal} / \mathrm{g} \rrbracket$ & 3.4 & 3.7 & 3.7 & 3.7 & 3.7 \\
\hline Protein $₫ \% \bigotimes$ & 19.4 & 19.4 & 19.4 & 19.4 & 19.4 \\
\hline Carbohydrates $₫ \% \rrbracket$ & 80.6 & 63.6 & 63.6 & 63.6 & 63.6 \\
\hline Fat $\otimes \% \bigotimes$ & 0 & 16.9 & 16.9 & 16.9 & 16.9 \\
\hline
\end{tabular}

Table 3 Effects of dietary fat on rat organ index

\begin{tabular}{|lllll|}
\hline Project & heart & liver & spleen & kidney \\
\hline WZ & $0.37 \pm 0.03^{\mathrm{a}}$ & $2.53 \pm 0.09^{\mathrm{bc}}$ & $0.20 \pm 0.01^{\mathrm{a}}$ & $0.63 \pm 0.03^{\mathrm{a}}$ \\
\hline SY & $0.35 \pm 0.03^{\mathrm{a}}$ & $2.58 \pm 0.06^{\mathrm{ab}}$ & $0.21 \pm 0.02^{\mathrm{a}}$ & $0.64 \pm 0.06^{\mathrm{a}}$ \\
\hline JL & $0.38 \pm 0.04^{\mathrm{a}}$ & $2.68 \pm 0.12^{\mathrm{a}}$ & $0.20 \pm 0.01^{\mathrm{a}}$ & $0.67 \pm 0.04^{\mathrm{a}}$ \\
\hline YY & $0.35 \pm 0.03^{\mathrm{a}}$ & $2.45 \pm 0.09^{\mathrm{c}}$ & $0.21 \pm 0.01^{\mathrm{a}}$ & $0.65 \pm 0.05^{\mathrm{a}}$ \\
\hline DY & $0.37 \pm 0.05^{\mathrm{a}}$ & $2.57 \pm 0.12^{\mathrm{bc}}$ & $0.21 \pm 0.02^{\mathrm{a}}$ & $0.65 \pm 0.02^{\mathrm{a}}$ \\
\hline
\end{tabular}

Different letters on the shoulder in the same column indicate significant difference in organ index at the 0.05 significant level 
Table 4 Effects of dietary fat on blood biochemical indices (mmol/L, $n=8, x \pm s$ )

\begin{tabular}{|llllll|}
\hline Project & GLU & TC & TG & HDL-C & LDL-C \\
\hline WZ & $4.44 \pm 0.60^{\mathrm{bc}}$ & $2.48 \pm 0.27^{\mathrm{b}}$ & $1.04 \pm 0.08^{\mathrm{b}}$ & $0.85 \pm 0.18^{\mathrm{a}}$ & $0.73 \pm 0.08^{\mathrm{ab}}$ \\
\hline SY & $5.06 \pm 0.51^{\mathrm{ab}}$ & $2.81 \pm 0.35^{\mathrm{ab}}$ & $1.50 \pm 0.26^{\mathrm{a}}$ & $0.66 \pm 0.09^{\mathrm{b}}$ & $0.71 \pm 0.17^{\mathrm{abc}}$ \\
\hline $\mathrm{JL}$ & $5.25 \pm 0.43^{\mathrm{a}}$ & $3.11 \pm 0.39^{\mathrm{a}}$ & $1.52 \pm 0.29^{\mathrm{a}}$ & $0.61 \pm 0.12^{\mathrm{b}}$ & $0.75 \pm 0.09^{\mathrm{a}}$ \\
\hline YY & $4.07 \pm 0.52^{\mathrm{c}}$ & $1.97 \pm 0.31^{\mathrm{c}}$ & $1.21 \pm 0.24^{\mathrm{b}}$ & $0.59 \pm 0.16^{\mathrm{b}}$ & $0.60 \pm 0.15^{\mathrm{bc}}$ \\
\hline DY & $4.77 \pm 0.65^{\mathrm{ab}}$ & $2.56 \pm 0.34^{\mathrm{b}}$ & $1.48 \pm 0.30^{\mathrm{a}}$ & $0.88 \pm 0.18 \mathrm{a}$ & $0.59 \pm 0.07^{\mathrm{c}}$ \\
\hline
\end{tabular}

Different letters on the shoulder in the same column indicate significant difference in blood biochemical indices at the 0.05 significant level

\section{Table 5 Effect of dietary fat on morphological structure of jejunum in rats}

\begin{tabular}{|llllll|}
\hline Project & WZ & SY & JL & YY & DY \\
$\begin{array}{l}\text { villus } \\
\text { height }(\mu \mathrm{m})\end{array}$ & $821.76 \pm 76.88^{\mathrm{d}}$ & $1078.80 \pm 43.50^{\mathrm{ab}}$ & $1101.18 \pm 55.01^{\mathrm{a}}$ & $990.67 \pm 103.25^{\mathrm{bc}}$ & $925.78 \pm 60.66^{\mathrm{c}}$ \\
\hline $\begin{array}{l}\text { crypt } \\
\text { depth }(\mu \mathrm{m})\end{array}$ & $254.44 \pm 13.68^{\mathrm{d}}$ & $293.48 \pm 10.12^{\mathrm{ab}}$ & $303.94 \pm 10.71^{\mathrm{a}}$ & $271.42 \pm 21.71^{\mathrm{cd}}$ & $279.48 \pm 11.40^{\mathrm{bc}}$ \\
$\begin{array}{l}\text { wall } \\
\text { thickness }(\mu \mathrm{m})\end{array}$ & $202.80 \pm 15.71^{\mathrm{c}}$ & $277.36 \pm 19.71^{\mathrm{a}}$ & $279.55 \pm 28.56^{\mathrm{a}}$ & $242.86 \pm 27.96^{\mathrm{b}}$ & $256.15 \pm 26.55^{\mathrm{ab}}$ \\
\hline $\begin{array}{l}\text { villus height / } \\
\text { crypt depth }\end{array}$ & $3.23 \pm 0.26^{\mathrm{c}}$ & $3.68 \pm 0.23^{\mathrm{a}}$ & $3.62 \pm 0.15^{\mathrm{ab}}$ & $3.66 \pm 0.36^{\mathrm{a}}$ & $3.31 \pm 0.18^{\mathrm{bc}}$ \\
\hline
\end{tabular}

Different letters on the shoulder in the same column indicate significant difference in morphological structure of jejunum at the 0.05 significant level

Table 6 Diversity index 


\begin{tabular}{|lllll|}
\hline Sample Name & Chao1 & ACE & Shannon & Simpson \\
\hline WZ & $1799.84 \pm 407.96^{\mathrm{a}}$ & $1867.58 \pm 193.98^{\mathrm{a}}$ & $8.32 \pm 0.39^{\mathrm{a}}$ & $0.98 \pm 0.00^{\mathrm{a}}$ \\
\hline SY & $2051.61 \pm 463.54^{\mathrm{a}}$ & $2159.56 \pm 735.43^{\mathrm{a}}$ & $8.34 \pm 0.31^{\mathrm{a}}$ & $0.98 \pm 0.01^{\mathrm{a}}$ \\
\hline JL & $1706.45 \pm 156.80^{\mathrm{a}}$ & $1858.77 \pm 401.48^{\mathrm{a}}$ & $8.45 \pm 0.27^{\mathrm{a}}$ & $0.99 \pm 0.00^{\mathrm{a}}$ \\
\hline YY & $1937.37 \pm 523.08^{\mathrm{a}}$ & $2090.81 \pm 432.75^{\mathrm{a}}$ & $8.41 \pm 0.30^{\mathrm{a}}$ & $0.98 \pm 0.01^{\mathrm{a}}$ \\
\hline DY & $1991.97 \pm 590.71^{\mathrm{a}}$ & $1940.17 \pm 521.48^{\mathrm{a}}$ & $8.18 \pm 0.13^{\mathrm{a}}$ & $0.98 \pm 0.00^{\mathrm{a}}$ \\
\hline
\end{tabular}

Different letters on the shoulder in the same column indicate significant difference in diversity index at the 0.05 significant level

Table7 Differences in gut microbiota at the level of phylum in each group

\begin{tabular}{|llll|}
\hline Sample Name & Bacteroidetes $\ \otimes$ & Firmicutes $\ \% \square$ & Firmicutes / Bacteroidetes \\
\hline WZ & $48.00 \pm 3.09^{\mathrm{b}}$ & $44.11 \pm 4.99^{\mathrm{a}}$ & $0.92 \pm 0.14^{\mathrm{a}}$ \\
\hline SY & $54.50 \pm 4.31^{\mathrm{ab}}$ & $42.48 \pm 5.59^{\mathrm{a}}$ & $0.79 \pm 0.16^{\mathrm{ab}}$ \\
\hline JL & $51.20 \pm 8.42^{\mathrm{ab}}$ & $43.76 \pm 4.14^{\mathrm{a}}$ & $0.88 \pm 0.24^{\mathrm{a}}$ \\
\hline YY & $67.74 \pm 10.29^{\mathrm{a}}$ & $29.09 \pm 8.44^{\mathrm{b}}$ & $0.44 \pm 0.19^{\mathrm{b}}$ \\
\hline DY & $54.93 \pm 12.66^{\mathrm{ab}}$ & $37.65 \pm 7.74^{\mathrm{ab}}$ & $0.73 \pm 0.27^{\mathrm{ab}}$ \\
\hline
\end{tabular}

Different letters on the shoulder in the same column indicate significant difference in the level of phylum at the 0.05 significant level

Table 8 Differences in gut microbiota at the level of family in each group

\begin{tabular}{|c|c|c|c|c|}
\hline Sample Name & Bacteroidaceae $\varangle \% \rrbracket$ & S24-7凶\%区 & Ruminococcaceae $\varangle \% \bigotimes$ & Lachnospiraceae $₫ \% \rrbracket$ \\
\hline WZ & $26.67 \pm 2.55^{a b}$ & $16.06 \pm 1.54^{c}$ & $19.82 \pm 0.47^{a}$ & $4.85 \pm 0.47^{a}$ \\
\hline SY & $29.95 \pm 7.34^{a}$ & $20.49 \pm 6.58^{b c}$ & $17.79 \pm 5.45^{a}$ & $3.77 \pm 0.74^{a}$ \\
\hline$J L$ & $14.14 \pm 6.90^{b}$ & $28.72 \pm 4.74^{a}$ & $19.15 \pm 6.91^{a}$ & $3.93 \pm 1.50^{a}$ \\
\hline YY & $24.57 \pm 7.21^{\mathrm{ab}}$ & $25.98 \pm 3.13^{a b}$ & $12.60 \pm 3.89^{a}$ & $4.42 \pm 0.98^{a}$ \\
\hline DY & $27.05 \pm 11.59^{\mathrm{ab}}$ & $24.50 \pm 1.78^{a b}$ & $16.43 \pm 6.99^{a}$ & $4.65 \pm 0.27^{a}$ \\
\hline
\end{tabular}

Different letters on the shoulder in the same column indicate significant difference in the level of family at the 0.05 significant level 


\section{Figures}

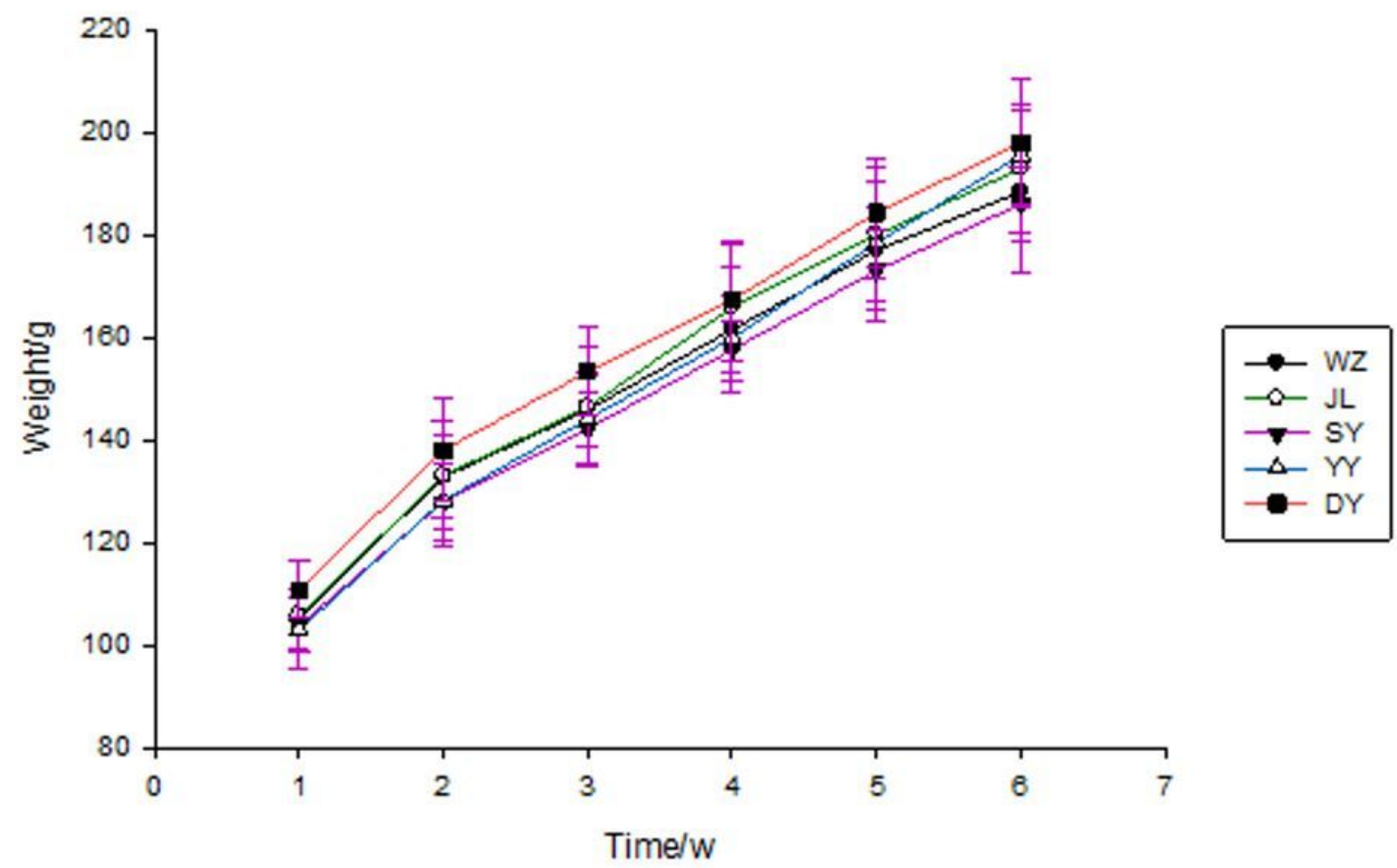

Figure 1

Effect of dietary fat on body weight in rats 
WZ

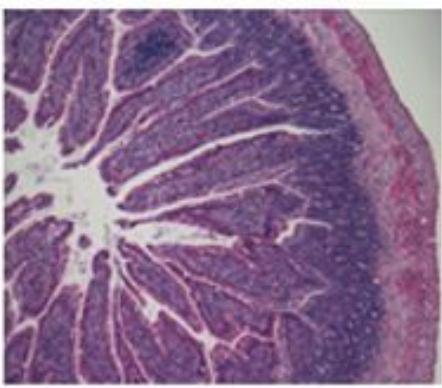

YY

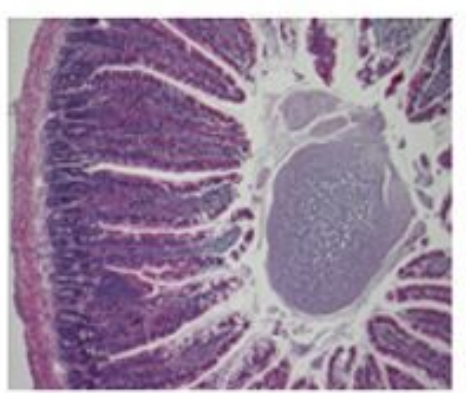

SY

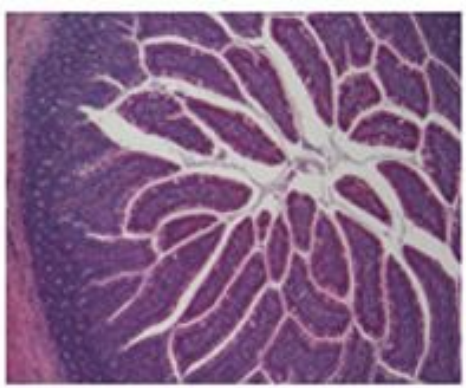

DY

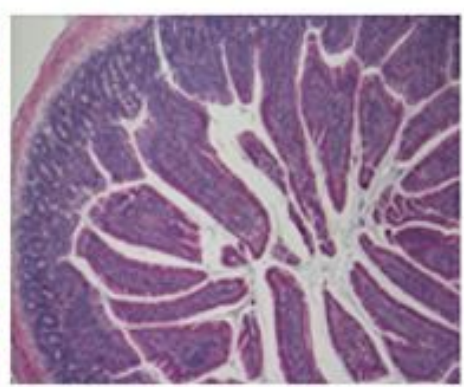

J

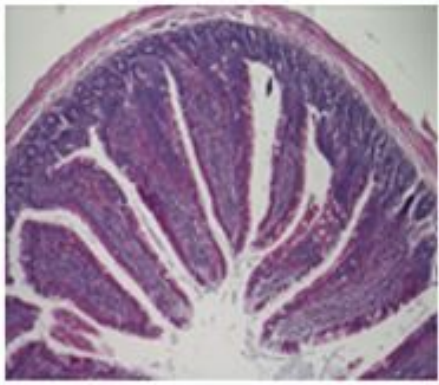

\section{Figure 2}

H\&E-stained of dietary fat intake for 6 weeks on the morphological structure of jejunum in rats 


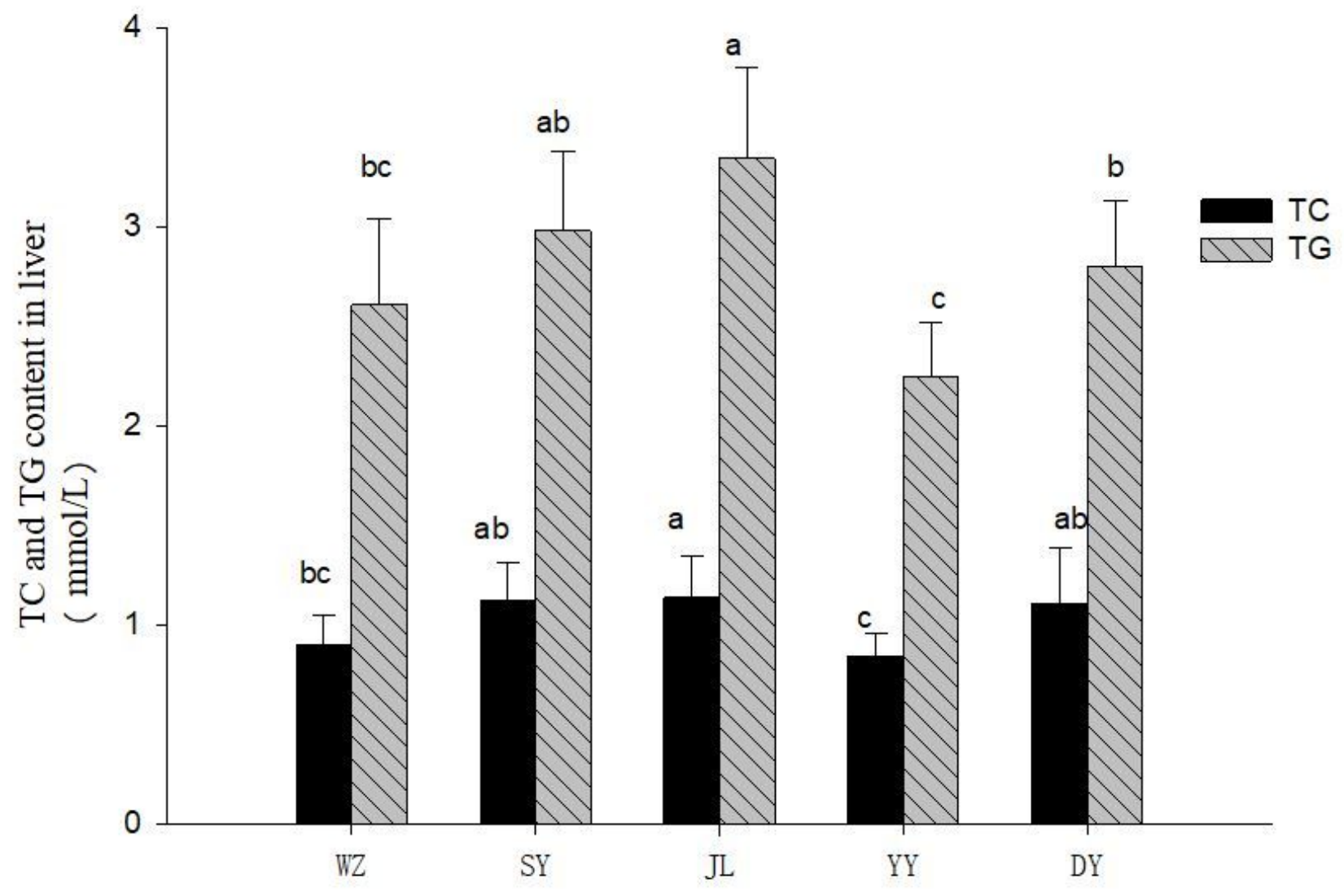

Figure 3

Content of cholesterol and triglyceride in rat liver Different superscript letters indicate that the contents of TC and TG in liver are significantly different at 0.05 significant level 


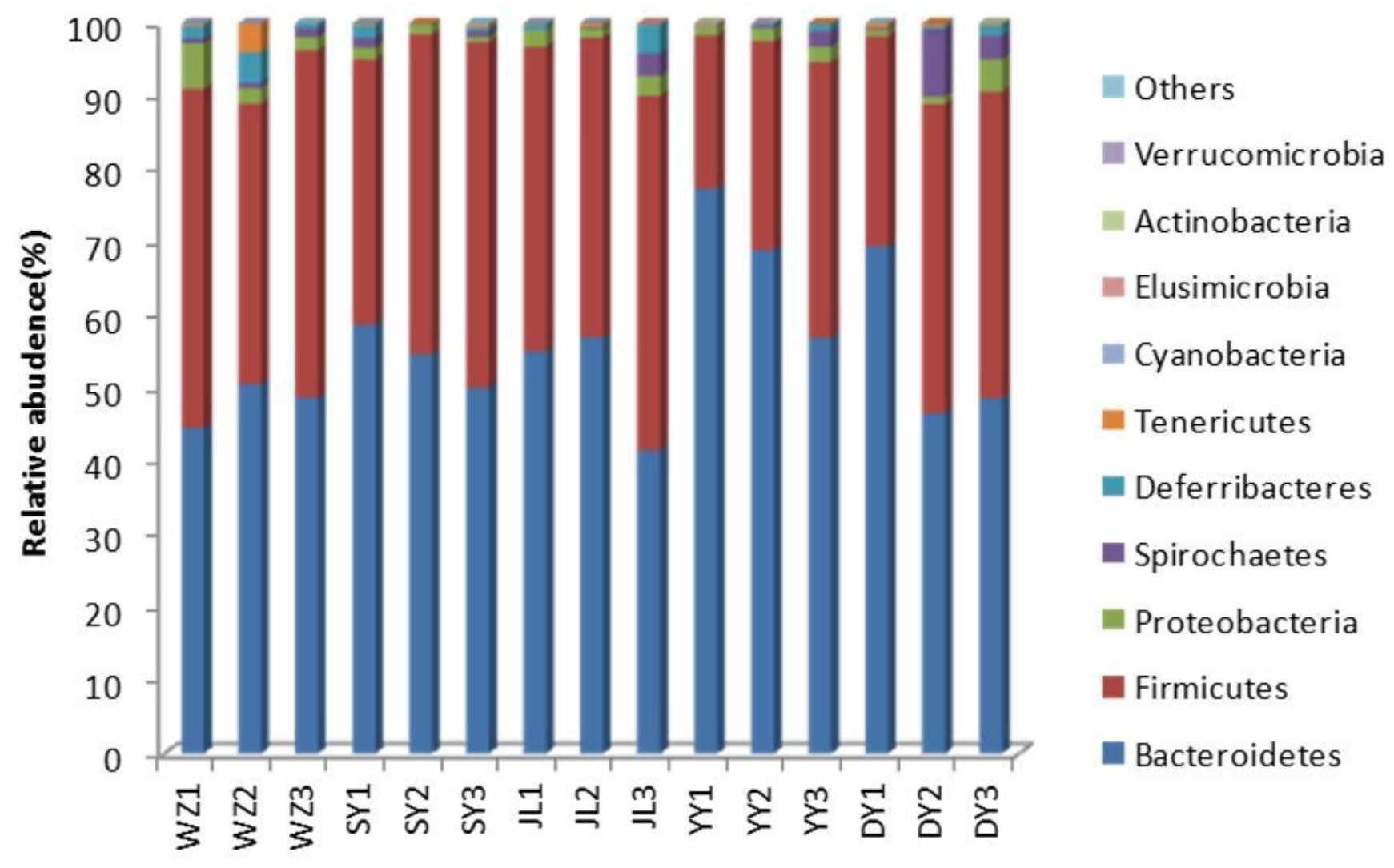

Figure 4

Effect of dietary fat on the phylum level of intestinal flora in rats 


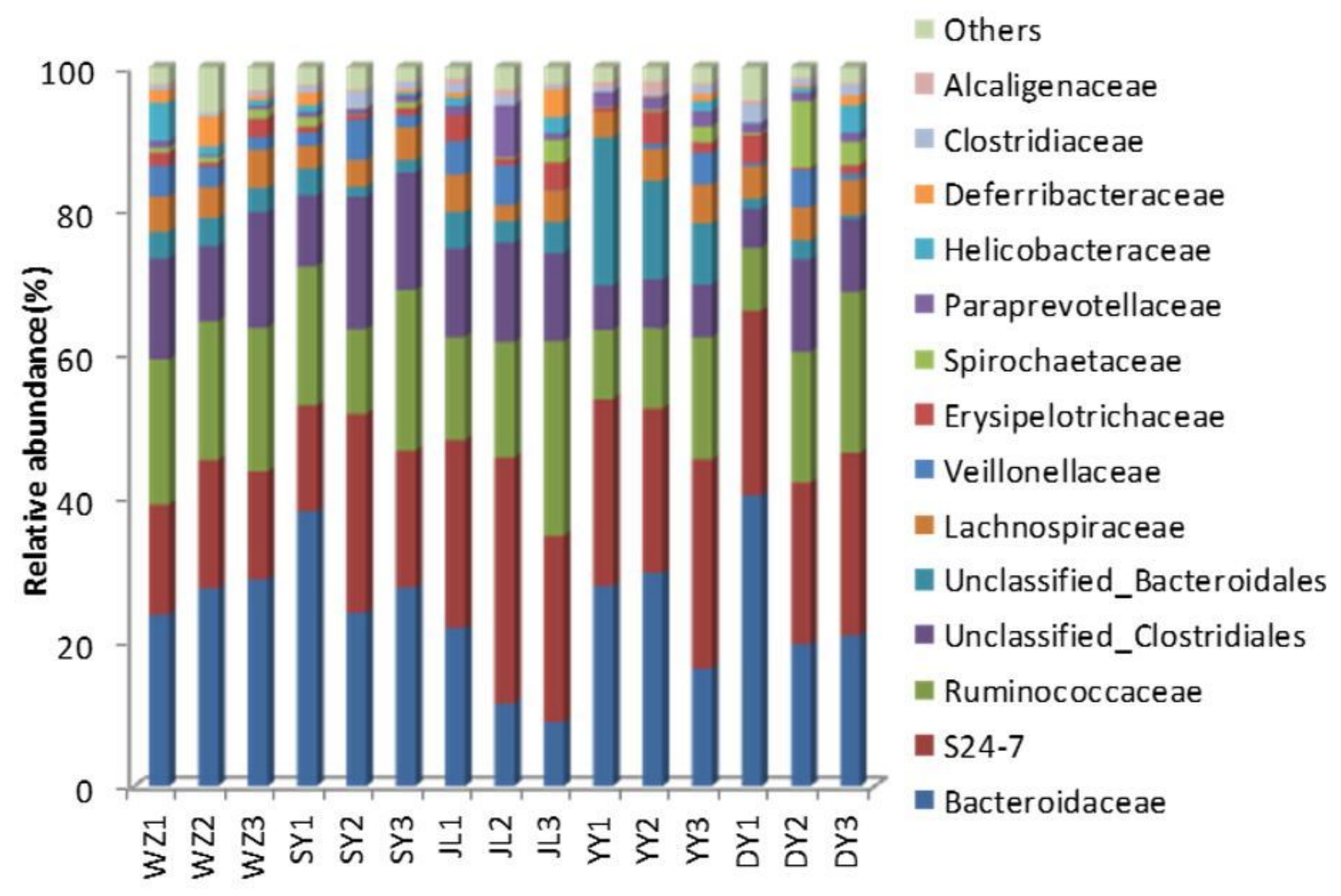

Figure 5

Effect of dietary fat on the family level of intestinal flora in rats 